\title{
Sustainability of healthcare professionals' adherence to clinical practice guidelines in primary care
}

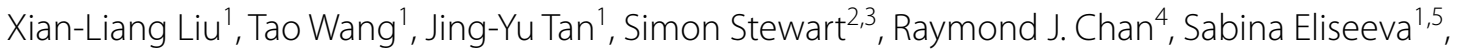
Mary Janice Polotan ${ }^{1,5}$ and Isabella Zhao ${ }^{1,6^{*}}$

\begin{abstract}
Background: Sustainability of adherence to clinical practice guidelines (CPGs) represents an important indicator of the successful implementation in the primary care setting.

Aim: To explore the sustainability of primary care providers' adherence to CPGs after receiving planned guideline implementation strategies, activities, or programmes.

Methods: Cochrane Central Register of Controlled Trials (CENTRAL); Cumulative Index to Nursing and Allied Health Literature (CINAHL); EMBase; Joanna Briggs Institute; Journals@Ovid; Medline; PsycolNFO; PubMed, and Web of Science were searched from January 2000 through May 2021 to identify relevant studies. Studies evaluating the sustainability of primary care providers' (PCPs') adherence to CPGs in primary care after any planned guideline implementation strategies, activities, or programmes were included. Two reviewers extracted data from the included studies and assessed methodological quality independently. Narrative synthesis of the findings was conducted.

Results: Eleven studies were included. These studies evaluated the sustainability of adherence to CPGs related to drug prescribing, disease management, cancer screening, and hand hygiene in primary care. Educational outreach visits, teaching sessions, reminders, audit and feedback, and printed materials were utilized in the included studies as guideline implementation strategies. None of the included studies utilized purpose-designed measurements to evaluate the extent of sustainability. Three studies showed positive sustainability results, three studies showed mixed sustainability results, and four studies reported no significant changes in the sustainability of adherence to CPGs. Overall, it was difficult to quantify the extent to which CPG-based healthcare behaviours were fully sustained based on the variety of results reported in the included studies.
\end{abstract}

Conclusion: Current guideline implementation strategies may potentially improve the sustainability of PCPs' adherence to CPGs. However, the literature reveals a limited body of evidence for any given guideline implementation strategy. Further research, including the development of a validated purpose-designed sustainability tool, is required to address this important clinical issue.

Trial registration: The study protocol has been registered at PROSPERO (No. CRD42021259748).

Keywords: Sustainability, Healthcare professionals, Adherence, Clinical practice guidelines, Primary care

\footnotetext{
*Correspondence: isabella.zhao@qut.edu.au

${ }^{6}$ Cancer \& Palliative Care Outcomes Centre, Faculty of Health,

Queensland University of Technology, 60 Musk Avenue, Kelvin Grove, QLD

4059, Australia

Full list of author information is available at the end of the article
}

(C) The Author(s) 2022. Open Access This article is licensed under a Creative Commons Attribution 4.0 International License, which permits use, sharing, adaptation, distribution and reproduction in any medium or format, as long as you give appropriate credit to the original author(s) and the source, provide a link to the Creative Commons licence, and indicate if changes were made. The images or other third party material in this article are included in the article's Creative Commons licence, unless indicated otherwise in a credit line to the material. If material is not included in the article's Creative Commons licence and your intended use is not permitted by statutory regulation or exceeds the permitted use, you will need to obtain permission directly from the copyright holder. To view a copy of this licence, visit http://creativecommons.org/licenses/by/4.0/. The Creative Commons Public Domain Dedication waiver (http://creativeco mmons.org/publicdomain/zero/1.0/) applies to the data made available in this article, unless otherwise stated in a credit line to the data. 


\section{Contributions to the literature}

- Current guideline implementation strategies may potentially improve the sustainability of primary care providers' adherence to clinical practice guidelines;

- No structured evaluation methods or purposedesigned tools were utilized to assess the healthcare professionals' sustainability levels.

- Maximum effort should be taken to ensure the longterm continuation of the implementation by planning the sustainability of adherence to clinical practice guidelines carefully and adopting a multipronged strategic approach.

\section{Background}

Clinical practice guidelines (CPGs) are evidence-based and systematically developed summaries and recommendations to assist healthcare professionals and patients in the process of healthcare decision-making [1]. CPGs can facilitate translation of up-to-date scientific research knowledge into practice and optimise care practices and outcomes for patients and their families [2]. When CPGs are adhered to, healthcare processes structures outcomes improve in primary care settings [3-5]. However, previous studies have shown that non-compliance of CPGs is as high as $70 \%$ in healthcare and occurs across most disciplines [6], including primary care [7]. Even in situations where there is CPG uptake, healthcare professionals may return to established clinical routine and practices, demonstrating and, therefore, have difficulty sustaining the successful implementation of CPGs in practice over a long period of time [6]. Strategies and activities undertaken beyond implementation to sustain CPG uptake are often inadequate [8]. Therefore, although CPGs are available and accessible to all healthcare professionals in primary care, the quality of healthcare services delivered to patients continues to vary $[9,10]$.

Implementation science refers to "...the scientific study of methods to promote the systematic uptake of research findings and other evidence-based practice into routine practice and, hence, to improve the quality and effectiveness of health services. (page 1)" [11] Implementation science tests the contextual factors affecting uptake and use of a clinical innovation, including sustainability, feasibility and fidelity [12]. Sustainability is a key outcome and priority quality indicator in implementation science. It refers to the extent to which a successful practice or programme is maintained as a clinical routine until it reaches obsolescence [13]. "Capability of being maintained at a certain rate or level (p. 1580)" is the simplest definition of sustainability [14]. Sustainability is often considered a result of maintaining health benefits or activities (e.g., cancer medication use and education) [13], and outcomes related to the implementation process (e.g., increased rates in continued use of the evidence-based innovations) [15]. The successful implementation of and adherence to CPGs in primary care is undeniably difficult. Several studies have explored the sustainability of innovations in primary care and reported that the innovations were not maintained after project funding had ended [16]. Overall, the sustainability of programmes were classified as either poor [17] or optimistic (sustainability score of $>55 / 100$ ) [18]. The sustainability of the implementation of CPGs may require a well-structured process to ensure policies become fully integrated. Important determinants of success or failure in this context include the implementation process, staff, and organizational factors $[19,20]$. One previous review aimed to evaluate the sustainability of healthcare professionals' adherence to CPGs in all healthcare settings. It reported that structured approaches and methods for sustainability evaluations were lacking [8]. Further, only three of the 14 included studies in the previous systematic review focused on primary care, with mixed sustainability results identified in that setting [21-23]. Therefore, no definitive conclusion could be drawn about the effects of any of the implementation strategies for the sustainability of primary care professionals' adherence to CPGs [8]; this being one of the least studied and understood issues in implementation research.

Within the above context, the aim of this literature analysis was to explore the sustainability of healthcare professionals' adherence to CPGs after receiving planned guideline implementation strategies, activities, or programmes in primary care. Specifically, this review explored (1) the effectiveness of different activities and programmes that targeted healthcare professionals in primary care to improve the sustainability of adherence to CPGs; (2) the current sustainability of healthcare professionals' adherence to CPGs in primary care; (3) the evaluation methods for the sustainability of adherence to CPGs in primary care; and (4) directions for increasing the sustainability of healthcare professionals' adherence to CPGs in primary care in future.

\section{Methods}

The Preferred Reporting Items for Systematic Reviews and Meta-Analyses (PRISMA) checklist was utilized in determining the information and process required for this literature analysis [24]. The study protocol has been registered at PROSPERO (No. CRD42021259748). 


\section{Data sources}

\section{Electronic database search}

An electronic database search was performed in nine databases to locate eligible publications from January 2000 through May 2021, and the search was limited to the English language only. The research team developed tailored search strategies in consultation with a university librarian. The databases were: Cochrane Central Register of Controlled Trials (CENTRAL); Cumulative Index to Nursing and Allied Health Literature (CINAHL); EMBase; Joanna Briggs Institute; Journals@Ovid; Medline; PsycoINFO; PubMed, and Web of Science. The following registers for ongoing or completed trials were also searched: ClinicalTrials.gov (https://clinicaltrials.gov/) and the Australian New Zealand Clinical Trials Registry (http://www.anzctr.org.au/). Moreover, the National Guideline Clearinghouse (NGC), the National Institute for Health and Care Excellence (NICE) and Turning Research Into Practice (TRIP) were also searched as secondary resources. The electronic database search strategies are reported in Additional file 2, and MESH terms and keywords were utilized in the database search (see Table 1).

\section{Reference list search}

The reference lists of the included studies were searched using the ISI Web of Science for publications, which cites included studies.

\section{Inclusion criteria}

(1) Types of studies: Randomized controlled trials (RCTs), non-randomized studies, and before-after controlled studies.

(2) Types of participants: Healthcare professionals working in primary care settings, including general practitioners (GPs), practice nurses, allied health providers, therapists (e.g., physiotherapists, occupational therapists, music therapists, and speech and language therapists), dietitians, paramedics, and community healthcare workers. Primary care was defined as "the provision of integrated, accessible health care services by clinicians who are accountable for addressing a large majority of personal health care needs, developing a sustained partnership with patients, and practicing in the context of family and community" (p. 192) [25].

(3) Types of interventions: Any planned strategies, activities, or programmes (e.g., professional, organizational, and financial programmes) as part of a guideline implementation project that facilitated the sustainability of healthcare professionals' adherence to CPGs in primary care. The sustainability of healthcare professionals' adherence to CPGs was one of the study outcomes.

(4) Types of comparators for controls: Usual conventional practice in primary care or only passively received guidelines without any planned guideline implementation strategies, activities, or programmes.

(5) Types of outcome measures: Any objective and subjective measure of the sustainability of healthcare professionals' adherence to CPGs (e.g., how long a CPG was sustained, follow-up sustainability assessment, and self-reported performance in the sustainability period) were included. Sustainability was defined as "the continued use of program components and activities for the continued achievement of desirable program and population outcomes" (p 2060) [26]. Therefore, multiple sustainability measuring methods were included even though the publications or reports did not mention the "sustainability" in the text, for example, quality of drug prescribing and cancer screening rates in accordance with the CPGs. The assessment of sustainability required its successful implementation in part of a practice, programme, or service that was then sustained for at least six months follow-up

Table 1 MESH terms and keywords

\begin{tabular}{|c|c|c|}
\hline & Mesh Terms & Entry Terms, Key Words or Free Words \\
\hline Sustainability & "Guideline Adherence" & $\begin{array}{l}\text { "Sustainab*" or "Sustain" or "Adherence }{ }^{* "} \text { or "Compliance } \\
\text { "Maintenance" or }\end{array}$ \\
\hline Clinical practice guidelines & "Implementation Science" or "Guidelines" or "Consensus" & $\begin{array}{l}\text { "Guidelin*" or "Pathway*" or "Evidence-based Recommenda- } \\
\text { tion*" or "expert opinion*" }\end{array}$ \\
\hline Primary care & $\begin{array}{l}\text { "Primary Health Care" or "Physicians, Primary Care" or "Pri- } \\
\text { mary Care Nursing" or "Community Health Services" }\end{array}$ & $\begin{array}{l}\text { "Primary Care" or "Primary Healthcare" or "General Pract*" or } \\
\text { "Practice Nurs*" or "Community Healthcare" }\end{array}$ \\
\hline Health professionals & $\begin{array}{l}\text { "Health Personnel" or "General Practitioner" or "Nurses, Com- } \\
\text { munity Health" }\end{array}$ & $\begin{array}{l}\text { "Allied Health Provider" or "Allied Health Professional*" or } \\
\text { "Community Healthcare Worker" or "Healthcare Professional" } \\
\text { or "Health Professional*" or "Therapist" or "Dietitian" or } \\
\text { "Paramedics" }\end{array}$ \\
\hline
\end{tabular}


after the completion of the CPGs implementation strategies.

\section{Assessment of eligibility}

Based on the inclusion criteria, all the retrieved articles' titles and abstracts were assessed by the reviewers during the search process. After reading the titles and abstracts of all the retrieved articles, all duplicated articles were excluded by EndNote version X9 (Clarivate Analytics, London, United Kingdom). Two reviewers (XLL and TW) independently read the titles and abstracts of all the potentially relevant studies that were identified by the initial broad literature search. If information from the titles and abstracts was not clear, the full texts of the papers were retrieved for further assessment. Decisions to include a study in the review were made by two reviewers (XLL and TW) after appraisal of the full texts of all retrieved articles. Any doubts during this process were settled by discussion and, if necessary, with a third reviewer (JYT). All excluded full-text articles were given specific reasons why they were excluded, and a list of excluded papers was summarized.

\section{Assessment of the risk of bias in the included studies}

The methodological quality and risk of bias were evaluated for each of the included studies using the Effective Practice and Organization of Care (EPOC) "risk of bias" tool [27]. The risk of bias of RCTs, non-randomized studies, and controlled before-after studies were assessed using nine risk of bias criteria related to randomization, allocation concealment, baseline outcome measures, baseline characteristics, outcome assessment, incomplete data, contamination, selective reporting, and other risk of bias [27, 28]. The EPOC "risk of bias" tool provides instructions for making decisions about the nine specific criteria as high, unclear, or low risk (see Supplemental file 1) [27].

If the details were not available in the articles, additional information was collected by contacting the corresponding authors of the relevant articles or reviewing their previously published protocols and articles. Two reviewers (XLL and TW) evaluated the risk of bias of the included studies independently, and any doubts during this process were settled by consultation or discussion with a third reviewer (JYT).

\section{Data extraction}

Data from included studies were extracted independently by two reviewers (XLL and TW). The reviewers utilized a predefined data extraction form to extract data from each included article. The study designs, research settings, participant demographics, guideline implementation strategies/activities/programmes, comparisons, sustainability outcomes, and sustainability measurements were extracted. For missing or unclear information on the details of the included studies, attempts were made to contact the authors of the included studies or review their previously published protocols and articles to obtain additional information, if possible.

\section{Data analysis}

The variability in guideline implementation strategies, CPGs, and the sustainability outcomes precluded a metaanalysis. Descriptive analysis was used for the synthesis of findings, including a summary of the characteristics of the sustainability assessments and descriptions of the level of sustainability of healthcare professionals' adherence to CPGs in primary care after planned activities or programmes. Narrative subgroup analysis was conducted based on different sustainability outcomes, including sustainability of drug prescribing improvement, chronic disease management, cancer screening and hand hygiene practice. The sustainability results from the included studies were summarized descriptively for comparisons between the planned activities and programmes.

\section{Results}

\section{Selection of studies}

Figure 1 illustrates the number of publications identified at each step and the reasons for their exclusion. Overall, the systematic database search yielded 1057 potential records. Based on the applied eligibility criteria, 58 records appeared related to the research topic and required a further assessment of their full texts. However, 48 records were subsequently excluded at this step. In addition, this review also incorporated a search of key organizations (e.g., TRIP, NGC, and NICE), the reference lists of the included studies, and the ISI Web of Science for publications. The organization searches yielded 16 potential records, 15 of which were excluded because they did not meet the inclusion criteria. As a result, a total of 11 studies were included in this literature analysis [21-23, 29-36].

\section{Characteristics of the included studies}

Table 2 provides a summary of the 11 included studies. These comprise nine RCTs [22, 23, 30-36], one nonrandomized controlled trial [29], and one before-after study [21]. The included studies were conducted in the United Kingdom [21, 22, 30], the Netherlands [33, 36], the United States [23, 32], Belgium [29], Portugal [31], Japan [34], and Spain [35]. Six studies reported the funding sources of their studies, such as Diabetes UK, the National Health Institute, and Pfizer [22, 23, 30, 31, 33, 36]. Eight studies comprised a total of 1705 healthcare 


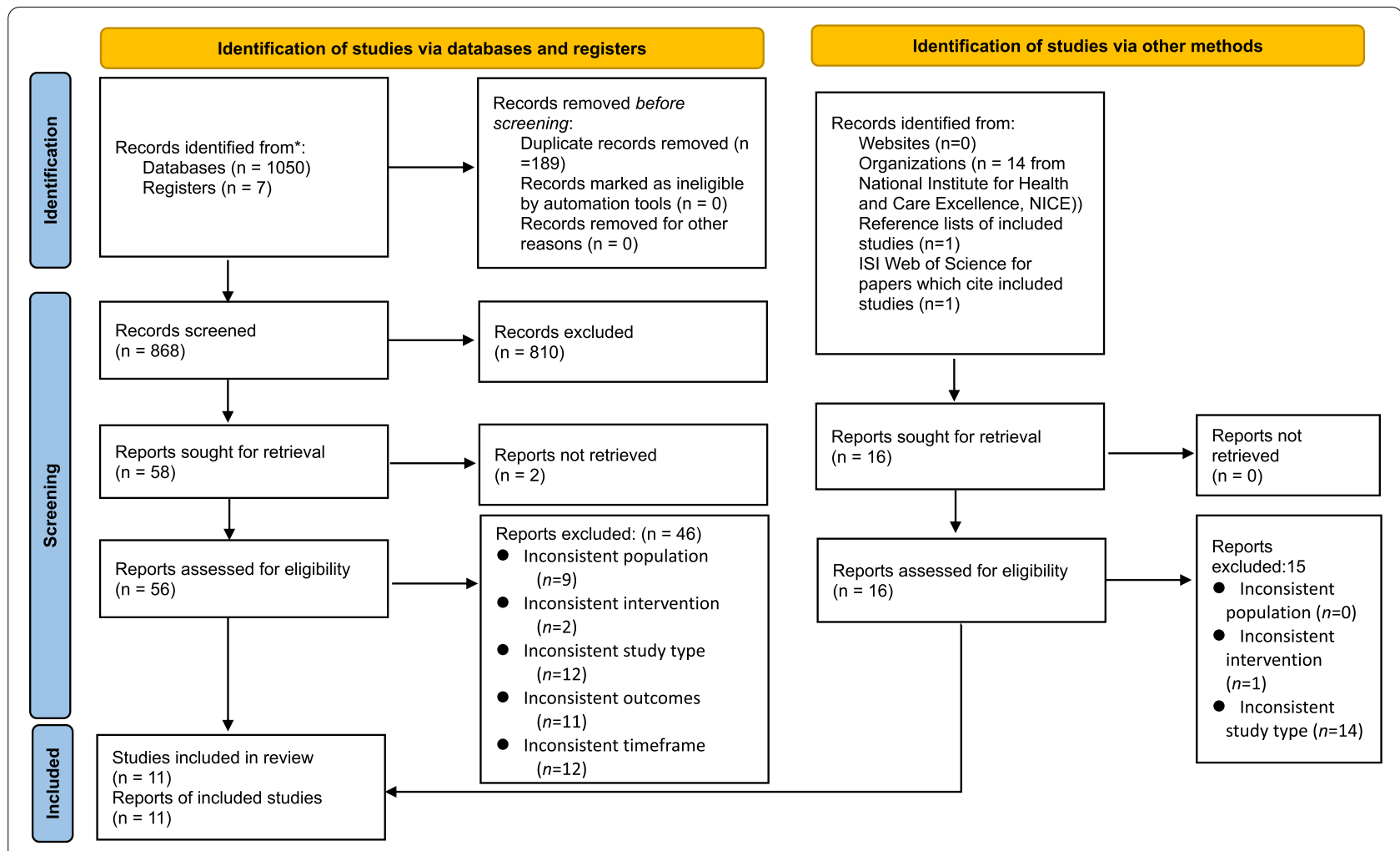

Fig. 1 PRISMA flow diagram for search results

professionals who completed the intervention and follow-up, while three of the included studies only reported the number of primary care centres that participated in the studies $(n=84$ [33], $n=28$ [22], and $n=1$ [21], respectively). Of the eight that reported number of participants, the average sample size of the included studies was 213 (range: 36-371). Most of the participants were GPs, and one included study recruited healthcare professionals from seven disciplines, including assistants in nursing, dental hygienists, GPs, midwives, nurses, odontostomatologists, and paediatricians [35]. The mean follow-up period was 16.1 months, ranging from six $[29,35]$ to 36 months [21].

The CPGs of focus widely varied. None of the included studies implemented the same CPG. Six studies targeted adherence to guidelines related to general practice drug prescribing $[21-23,31,33,36]$, whereas three studies aimed to improve adherence to guidelines related to disease management $[29,30,34]$ and one study each targeted improved cancer screening [32] and hand hygiene practice [35]. For the control groups, the same strategy was utilized for both the intervention group and the control group for different clinical topics (e.g., audit and feedback meetings) [36] or drug prescribing (e.g., educational outreach visits) [22] in two studies. The other included studies provided no active strategies, passive dissemination of guidelines, or implementation materials to the control group at the end of the study [23, 29-35].

\section{Guideline implementation strategies}

A variety of guideline implementation strategies were used in the included studies. Many combined more than two strategies to implement CPGs. These included educational outreach visits $[22,29-31,36]$, teaching sessions [32, 35], seminars [34], audit and feedback [23, $36]$, and reminders [29, 35]. Printed materials were provided in eight studies [21, 29-35]. Videos were utilized in two included studies to demonstrate practice-based examples, barriers, and solutions [30] and hand hygiene techniques [35]. After the first visit or teaching session, feedback of the performance was provided to the healthcare professionals in four included studies [22, 23, 33, 36]. For the duration of the guideline implementation strategies, educational outreach visits, teaching sessions, and seminars were 15 to $120 \mathrm{~min}$ in duration and were conducted in one to four sessions over one to six months [22, 23, 29-36]. Two studies utilized theories to develop their implementation strategies, such as the Social Cognitive Theory and the Health Action Process Approach [30, 32]. Consequently, no study applied an 


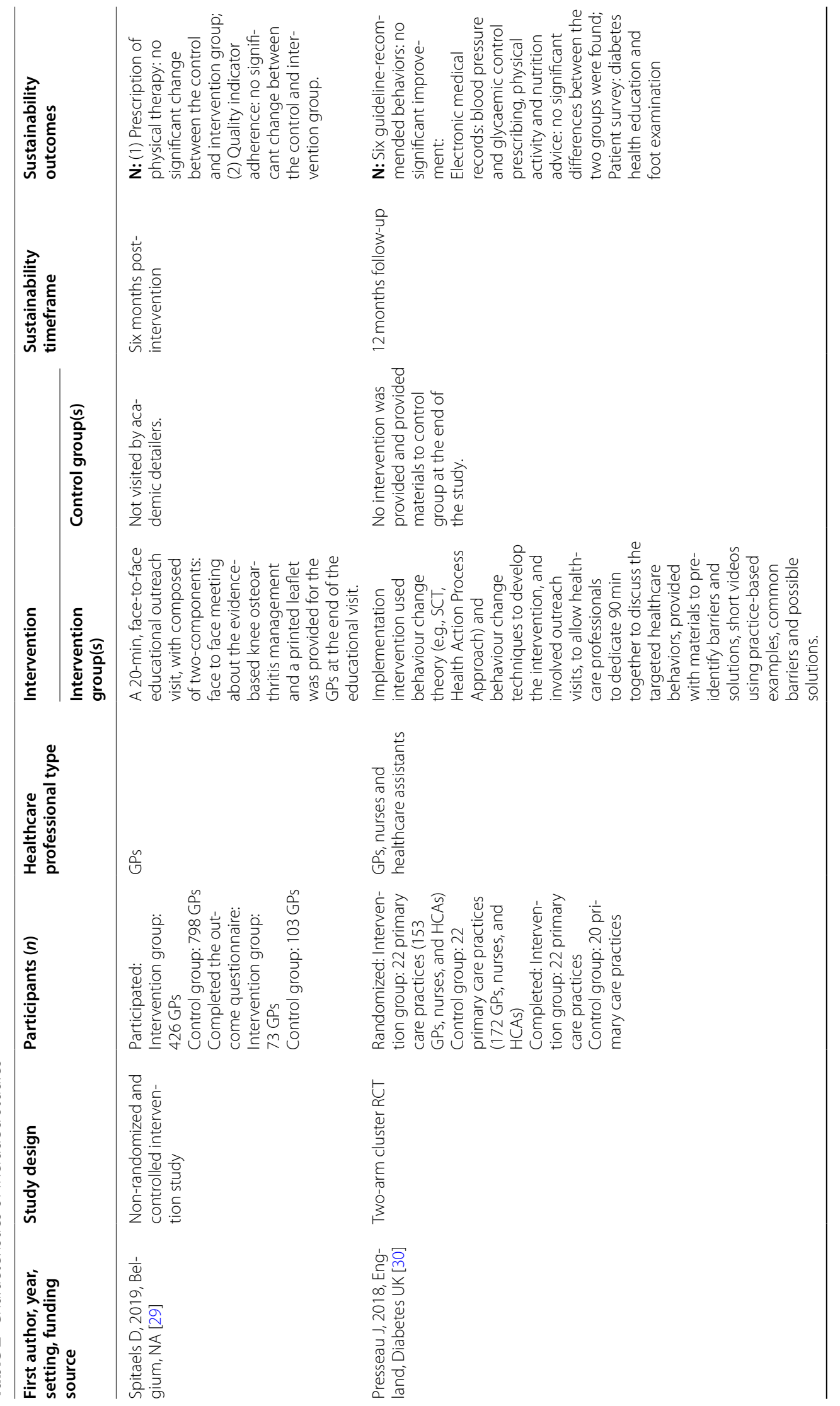




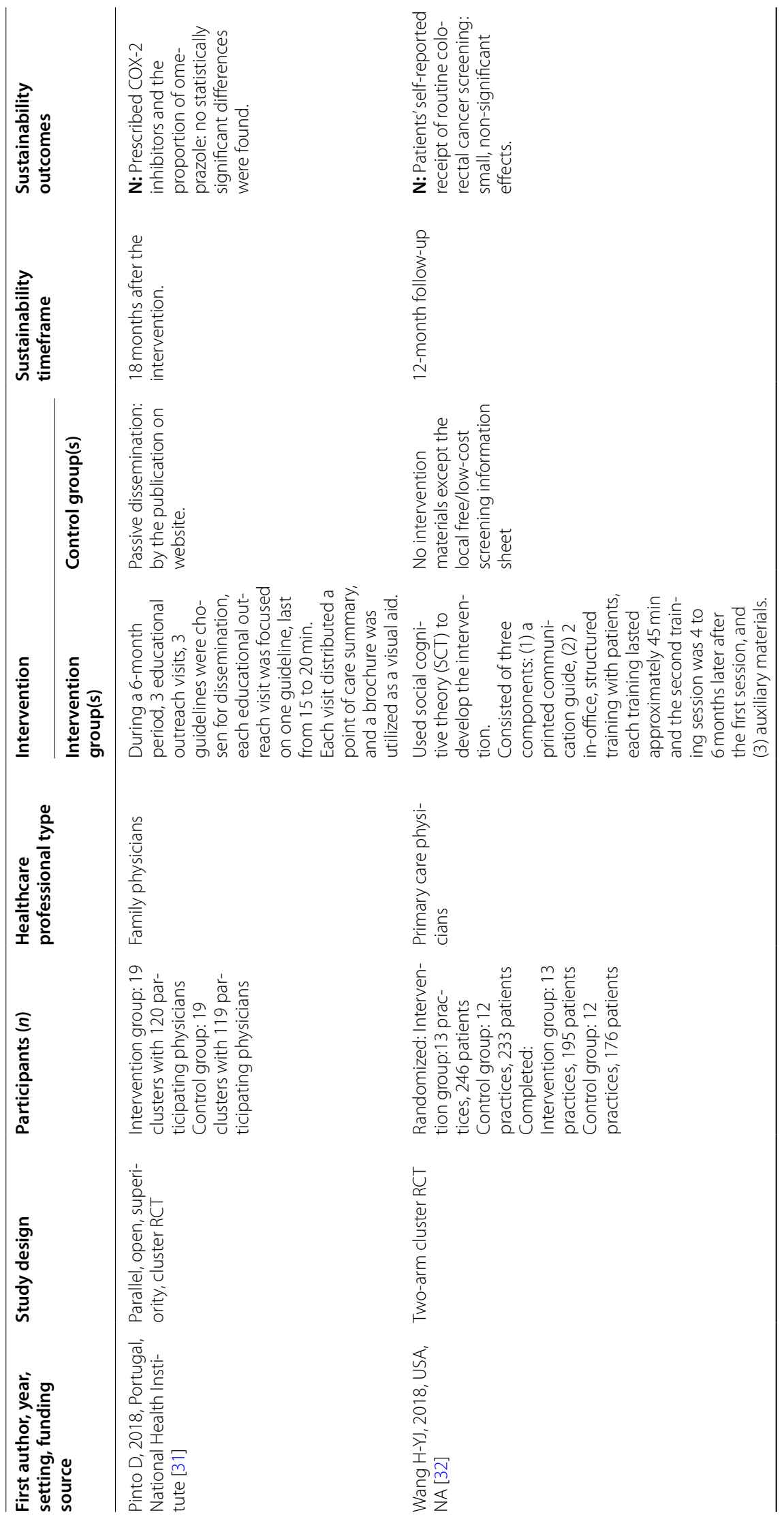




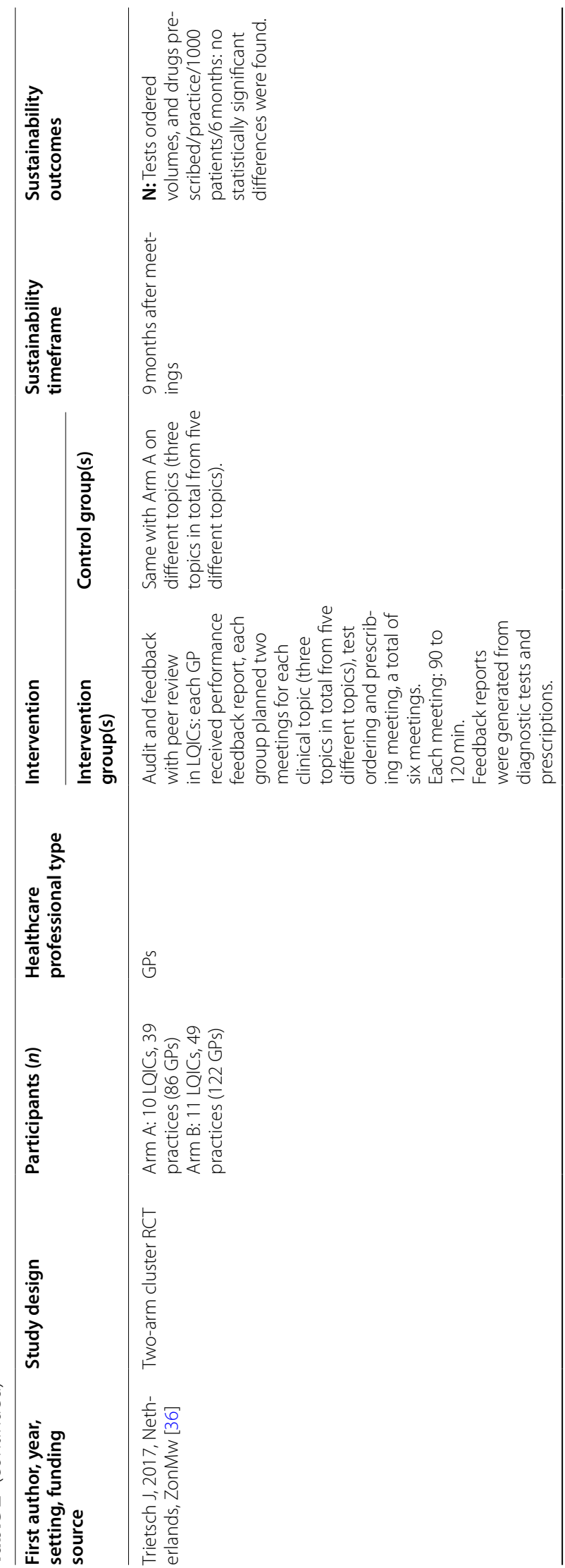




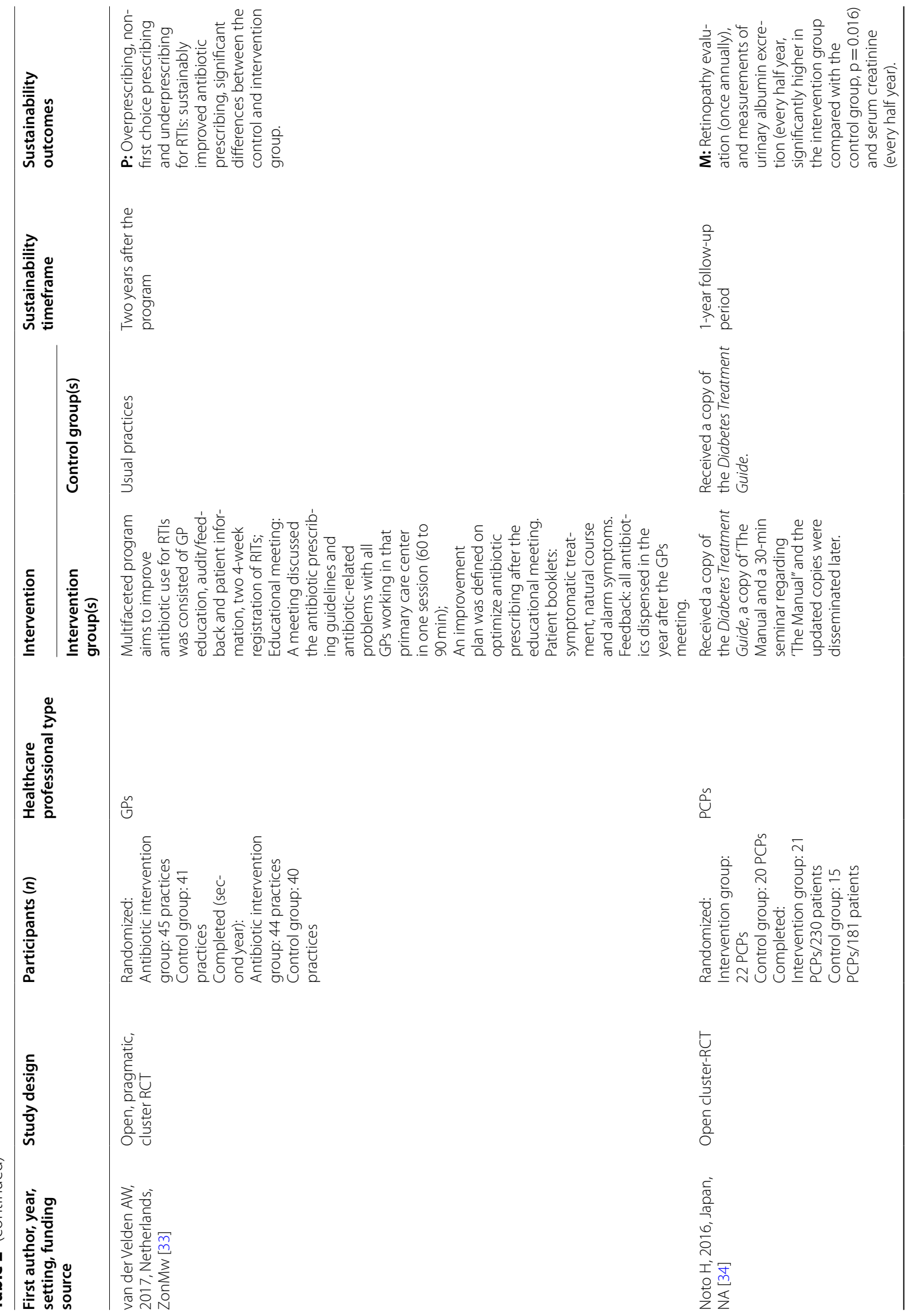




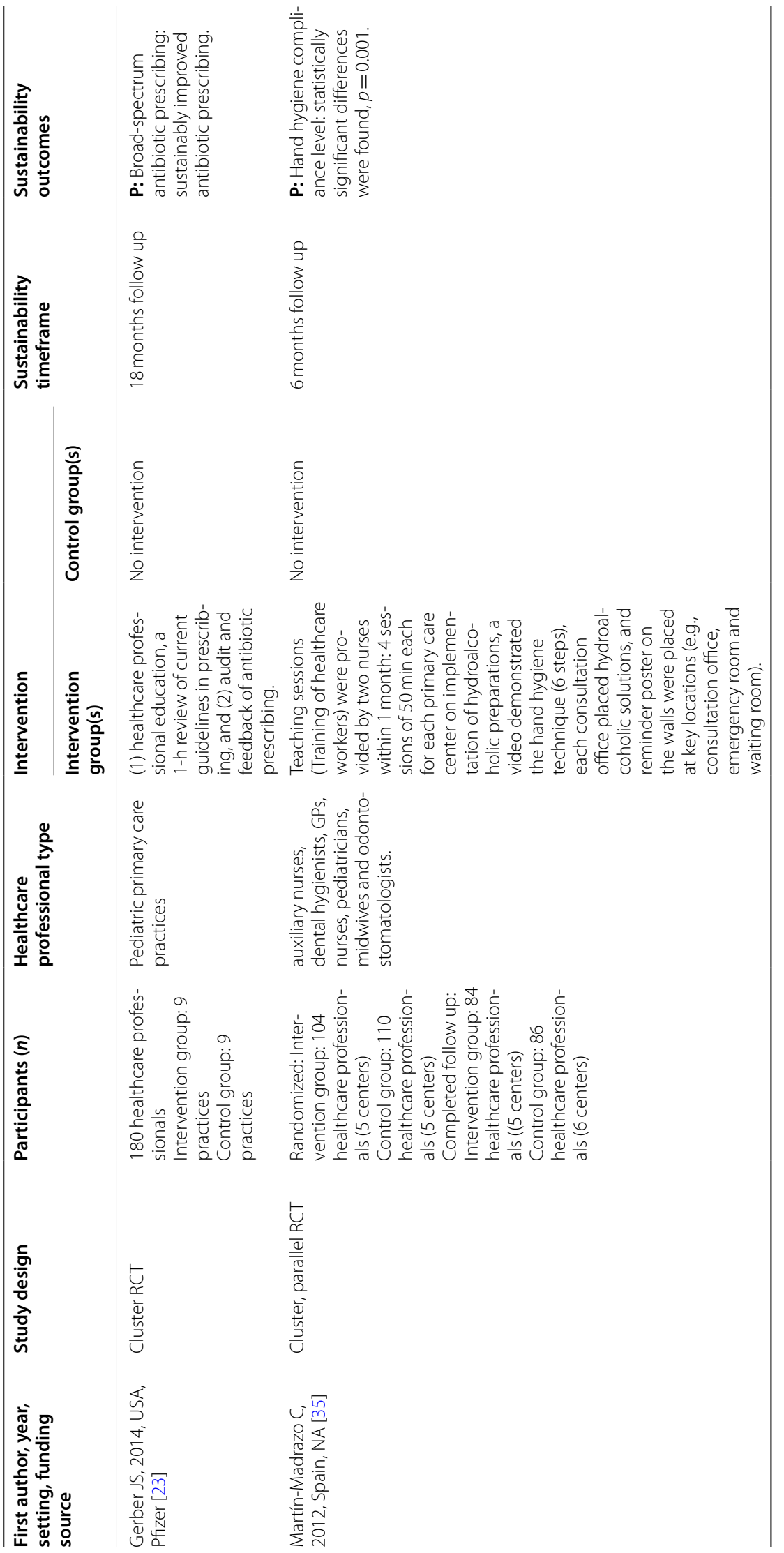




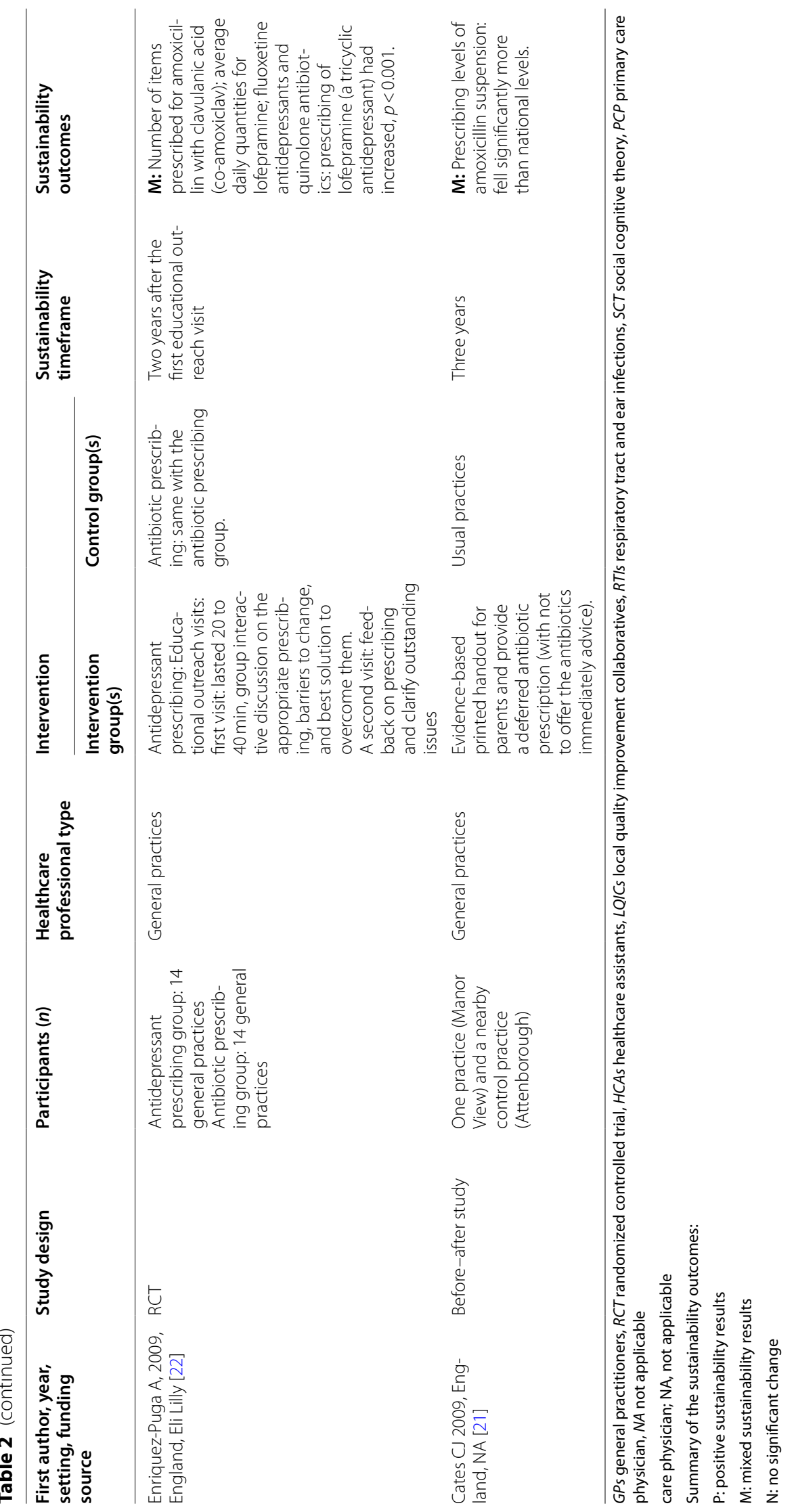


implementation framework or a sustainability-related theoretical framework.

\section{Sustainability evaluation methods}

None of the included studies utilized purpose-designed measurements or structural methods to evaluate sustainability levels. The most common strategy to evaluate sustainability was the analysis of routinely collected data from healthcare professionals. For example, drug prescribing was used in seven included studies [21-23, 30, 31, 33, 36]. Sustainability outcomes included guideline-recommended behaviours (e.g., blood pressure and glycaemic control prescribing and physical activity and nutrition advice) [30], disease management quality indicator adherence (e.g., referral for physical therapy, evaluation of retinopathy) [29, 34], cancer screening compliance [32], and hand hygiene compliance [35]. Seven included studies collected sustainability outcomes at multiple primary care centres using a cluster RCT design $[23,30-34,36]$.

\section{Methodological quality and risk of bias of the included studies}

Table 3 illustrates the risk of bias summary of each included study. Only 3 studies fulfilled six of the nine methodological quality criteria and were rated as having a relatively lower risk of bias (a higher score represented a lower risk of bias) [30, 31, 36], Seven studies fulfilled four or fewer criteria [21, 23, 29, 32-35]. Common forms of potential bias across all studies included a limited description of the approach of randomization, allocation concealment, missing data, blind outcome assessment, and protection against contamination. For example, although randomization was mentioned in nine RCTs, only four described the precise information of the random sequence generation process (low risk of bias) [22, 30, 31, 36]. Adequate allocation concealment was reported in three of these RCTs (low risk of bias) [30, 31, 36]. Limited information was used in the evaluation of the risk of bias in two studies as these two studies were published as a brief research report [21,23]. Three RCTs described and utilized intention to treat analysis (low risk of attrition bias) [31, 32, 35]. Critically, funnel plot analysis was not feasible in this review because of the scarcity of included studies.

\section{Descriptive analysis of sustainability outcomes Sustainability of drug prescribing improvement}

Six studies targeted sustainable improvements in the quality of drug prescribing in daily practice according to the specific recommendations of guidelines in primary care $[21-23,31,33,36]$. One trial showed no sustained effectiveness differences in drug prescribing in accordance with the guidelines between the intervention and the control groups at 18 months after the intervention (e.g., proportion of COX-2 inhibitors prescribed: $12.07 \%$ vs $13.08 \%, P=0.085$; proportion of omeprazole prescribed: $46.28 \%$ vs $47.15 \%, P=0.971$ ) [31]. Trietsch et al. utilized audit and feedback for three different practice topics, and the increase in inappropriate testing and prescribing behaviour was $20 \%$ in the intervention group and $66 \%$ in the control group at nine months after the meeting [36]. Overall, the study did not show a decrease in the volume of inappropriate test ordering and drug prescribing after nine months of the intervention; however, a lesser increase was found in the intervention group [36].

Table 3 Methodological quality assessment of included studies

\begin{tabular}{|c|c|c|c|c|c|c|c|c|c|c|}
\hline & Studies & Item 1 & Item 2 & Item 3 & Item 4 & Item 5 & Item 6 & Item 7 & Item 8 & Item 9 \\
\hline 1 & Spitaels D, 2019, Belgium [29] & High & Unclear & Low & High & Low & Unclear & Unclear & Low & Unclear \\
\hline 2 & Presseau J, 2018, England [30] & Low & Low & Low & Low & Low & Low & Low & Low & High \\
\hline 3 & Pinto D, 2018, Portugal [31] & Low & Low & Low & Low & Low & High & High & Low & High \\
\hline 4 & Wang H-YJ, 2018, USA [32] & Unclear & Unclear & Low & Low & Low & Unclear & Unclear & Low & Unclear \\
\hline 5 & Trietsch J, 2017, Netherlands [36] & Low & Low & Low & Low & Low & Low & High & Low & Unclear \\
\hline 6 & van der Velden AW, 2017, Netherlands [33] & Unclear & High & Low & Low & Unclear & Unclear & Unclear & Low & Unclear \\
\hline 7 & Noto H, 2016, Japan [34] & Unclear & Unclear & Low & High & Unclear & Low & Low & Low & High \\
\hline 8 & Gerber JS, 2014, USA [23] & Unclear & Unclear & Unclear & Unclear & Unclear & Unclear & Unclear & Unclear & Unclear \\
\hline 9 & Martín-Madrazo C, 2012, Spain [35] & Unclear & Unclear & Low & Low & Unclear & Unclear & Unclear & Low & Unclear \\
\hline 10 & Enriquez-Puga A, 2009, England [22] & Low & Unclear & Low & Low & Unclear & Low & Unclear & Low & Unclear \\
\hline 11 & Cates CJ 2009, England [21] & High & High & High & High & Unclear & Unclear & Unclear & Low & Unclear \\
\hline
\end{tabular}

Item 1 random sequence generation; Item 2 adequate concealment of allocation; Item 3 similar baseline outcome measures; Item 4 similar baseline characteristics; Item 5 blinding of outcome assessment; Item 6 adequately addressed incomplete outcome data; Item 7 adequate protection against contamination; Item 8 free from selective reporting; and Item 9 free of other risk of bias

Source: $h$ ttps://epoc.cochrane.org/resources/epoc-resources-review-authors

Low $=$ "Low risk", High ="High risk", Unclear="Unclear risk" 
Another study [22] aimed to improve the prescribing of selected antibiotics and antidepressants and reported that educational outreach visits showed no effect on prescribing quality, except that the prescribing of lofepramine (a tricyclic antidepressant) had increased. Educational outreach visits had a small, sustained effect on drug prescribing over the 24-month follow-up period, and prescribing lofepramine increased according to the guidelines, with the rate ratio $=2.85(P<0.001)$ [22]. With the aim of achieving sustainable effectiveness in antibiotic prescribing quality, van der Velden et al. [33] adopted a multifaceted implementation programme and reported significant improved antibiotic prescribing quality between the two groups in dispensed antibiotics per 1000 patients one year $(-7.6 \%$ vs $-0.4 \%, P=0.002)$ and two years $(-4.3 \%$ vs $+2.0 \%, P=0.015)$ after the intervention (decreased prescription indicates improvement). Similar results were reported for macrolides and amoxicillin/clavulanate prescribing, with the first year $-12.7 \%$ vs $+2.9 \%(P=0.001)$ and the second year $-7.8 \%$ vs $+6.7 \%(P=0.005)$ after the intervention [33]. Van der Velden et al. concluded that part of the multifaceted programme improvement was sustainable, as changes between the two groups were still present 24 months after the intervention [33]. During three-year follow-up, evidence-based patient handouts brought about a sustainable reduction in antibiotics prescribing for patients with acute otitis media [21]. Gerber et al. [23] reported similar results, as antibiotic prescribing decreased from 26.8 to $14.3 \%$ in the intervention group and decreased from 28.4 to $22.6 \%$ in the control group at 18 months after the intervention.

\section{Sustainability of chronic disease management}

Three studies aimed to improve the community management of diabetes [30, 34] and knee osteoarthritis [29]. Noto et al. conducted an RCT with an intervention arm that additionally provided a copy of The Standard Diabetes Manual and a 30-min seminar regarding The Standard Diabetes Manual compared with the control group, which received a copy of Diabetes Treatment Guide only [34]. The proportion of GPs who adhered to urinary albumin excretion measurement was significantly higher in the intervention arm (17.9\%) compared with the control arm (5.3\%) over a 12-month follow-up period $(P=0.016)$ [34]. Another study developed an intervention aimed at enhancing six guideline-recommended healthcare professional behaviours in type 2 diabetes management. Unfortunately, this intervention did not offer a significant improvement in any of these six behaviours at 12 months follow-up [30]. However, about $80 \%$ of the patients were examined for circulation and sensation in their feet, more than $70 \%$ of patients with a BMI $>30$ were provided personalized nutrition advice, and about $50 \%$ of the patients were prescribed additional therapy for blood pressure and personalized physical activity advice at 12 months follow-up. Overall, these behaviours' sustainability level was relatively high during the followup period [30]. In addition, educational outreach visits did not lead to significant changes in adherence to quality indicators for knee osteoarthritis management (e.g., referral for physical therapy) among GPs at six months after the outreach visits $(43.8 \%$ vs $31.3 \%, P=0.057)$ [29].

\section{Sustainability of cancer screening}

One cluster RCT evaluated an intervention targeted at training GPs to increase patients' colorectal cancer screening in primary care, and the intervention consisted of three components: a printed communication guide; two structured training sessions; and auxiliary materials [32]. At 12 months follow-up, the colorectal cancer screening rates were slightly higher in the intervention group (24.4\%) compared with those in the control group (17.7\%), however such difference was not statistically significant $(P=0.24)$ [32].

\section{Sustainability of hand hygiene practice}

One cluster RCT aimed to test a multimodal hand hygiene improvement programme for healthcare professionals (including assistants in nursing, dental hygienists, GPs, nurses, paediatricians, midwives, and odontostomatologists) for an improved hand hygiene compliance level in primary healthcare centres [35]. The multimodal intervention consisted of the implementation of hydroalcoholic solutions, teaching sessions, and reminder posters [35]. During a six-month follow-up period, the healthcare professionals in the intervention arm enhanced their hand hygiene compliance level by $21.6 \%$ compared with the control arm, but the hand hygiene compliance of the healthcare professionals in the intervention group did not significantly improve, and remained at $32.74 \%$ [35].

\section{Discussion}

This literature analysis identified 11 studies relevant to this important clinical topic. The duration of follow-up varied from six to 36 months. The results of the included studies showed that guideline implementation strategies (e.g., educational outreach visits, teaching sessions, reminders, and audit and feedback) potentially improve the sustainability of healthcare professionals' adherence to CPGs in drug prescribing, disease management (e.g., diabetes), and hand hygiene practice in primary care $[21,29,30,33,35]$. However, as there was a variety of implementation protocols and outcome measurements reported in the included studies, it was difficult to quantify the extent to which CPG-based healthcare 
behaviours were sustained, and none of the included studies could be utilized in a meta-analysis. No structured evaluation methods or purpose-designed tools were utilized to assess the healthcare professionals' sustainability levels. The included studies reported that achieving sustainable adherence to CPGs was a complex goal that was often hampered by practicalities and continued active efforts to sustain improvements $[21,23,30$, 31]. When designing a CPG implementation programme, maximum effort should be taken to ensure the long-term continuation of the implementation by planning the sustainability of adherence to CPGs carefully and adopting a multipronged strategic approach.

One relevant systematic review [8] identified 14 studies that aimed to improve the sustainability of healthcare professionals' adherence to CPGs in a wide range of medical care settings (acute care and primary care). Three studies included in that review were also considered in this analysis. The published review also reported that long-term adherence (more than one year after implementation) was not sustained in about half of the studies [8]. Moreover, it reported that no firm conclusions about the sustainability of healthcare professionals' adherence to CPGs in medical practice could be drawn based on the absence of a uniform definition, limited methodological rigour, and the mixed results of the studies [8]. Another systematic integrative review focused on the sustainability of healthcare system improvements, interventions, and programmes and reported that the body of literature was limited, with inconsistent definitions and measures of sustainability [37]. The findings of our literature analysis were consistent with these previous reviews.

According to the findings of our literature analysis, we concluded that the sustainability of healthcare professionals' adherence to CPGs in primary care was unsatisfactory, and that knowledge about structured approaches to sustaining adherence to CPGs among primary care professionals remains limited. Moreover, only two studies used theories or theoretical frameworks to design their implementation programmes [30, 32]. Healthcare professionals should be guided by suitable frameworks, models and theories (F/M/Ts) to advance sustainability in healthcare with an understanding of the factors that contribute to sustainability [38, 39]. Different F/M/ Ts have been used for establishing the theoretical based strategies to facilitate and sustain implementation programs [40], such as Dynamic Sustainability Framework (DSF) and NHS Sustainability Model (NHS SM) and Sustaining Organizational Change Framework (SOCF) [41]. The goals of the use of $\mathrm{F} / \mathrm{M} / \mathrm{Ts}$ in implementation programs including guiding the process of transferring updated evidence into healthcare practice, explaining influencing factors of implementation outcomes and evaluating implementation [42].

Conceptual and methodological limitations in measuring the outcomes of sustainability have been found. A related challenge is that conceptual frameworks with clear operational definitions or rigorous measures of sustainability are not often used [43], as was the case in most of the included studies. Measuring the sustainability of healthcare professionals' adherence to CPGs is complex, with relatively little attention paid to long-term maintenance [44]. Moore et al. described five elements for the assessment of sustainability, including a defined period of time and the continued delivery of an intervention and/ or maintenance of beneficial behaviour, meaning behavioural changes may evolve or adapt while continuing to produce benefits [45]. Other common terms and concepts covered by these studies included durability [23], persistence [31], follow-up [22, 30-32, 34, 36], and long term [31]. Trial evaluation periods provided clear, final evaluation timepoints, ranging from six months [29, 35] to the longest evaluations reported at three years [21]. The evaluation methods to assess sustainability levels of CPG adherence differed across the included studies, and none utilized validated purpose-designed tools. The most common form of evaluation was the analysis of routinely collected data from healthcare professionals.

\section{Quality of the evidence}

Overall, we found a limited body of evidence for any given guideline implementation strategy. The included studies did not provide sufficient evidence to determine the effectiveness of the interventions for improving the sustainability of healthcare professionals' adherence to CPGs in primary care. Less than one in three studies were rated as having a relatively lower risk of bias, so the levels of evidence in majority of the studies were downgraded because of the significant risk of bias. Seven studies used a cluster design, and the unit of randomization was the cluster (e.g., primary care centres), not the individual healthcare professional, so the baseline characteristics of the healthcare professionals were not comparable [22, 30, 32-36]. Moreover, none of the identified studies used validated purpose-designed tools to measure sustainability levels, and the most common evaluation approach used to measure sustainability was the analysis of routinely collected data from healthcare professionals. In addition, only six studies performed a sample size calculation to justify the included sample [29-31, $33,35,36]$. This literature analysis found a serious risk of bias; moreover, the small number of studies for each intervention and heterogeneous outcomes prevented us from drawing definitive conclusions. 


\section{Implications for further research and practice}

Based on this synthesis of the relevant literature, there is a clear indication for more rigorous studies to develop guideline implementation strategies to improve the sustainability of healthcare professionals' adherence to CPGs in primary care. While one or two included studies evaluated similar interventions and similar guidelines, we found insufficient data on which to base a conclusion on the most effective approaches or recommendations to address this key clinical issue. This includes identifying the key enablers and barriers to maintain the application of CPGs in the primary care setting. No included studies were identified from low-income countries. While this may be attributable to a relatively lower prominence of primary care in different healthcare systems, as well as the resource-intensive nature of designing and conducting implementation studies in low- and middle-income countries $[15,46]$ this is represents a key priority for future research.

The core implementation programmes of all the included studies included some type of educational approach (e.g., educational outreach visits, teaching sessions, and written materials) and reported that part of the effect was sustainable [21, 29, 30, 33, 35]. Even if educational strategies are an important element in the sustained process, theory-based implementation interventions may be worth further research, and behavioural change techniques or behavioural change models may help guide the development of these interventions. The standard definition and use of $\mathrm{F} / \mathrm{M} / \mathrm{Ts}$ should be developed to guide implementation program design to facilitate program long-term sustainability. Understanding the determinants of the sustainability of healthcare professionals' adherence to CPGs and including elements to address them may facilitate sustained benefits in healthcare services and patient outcomes over time. Moreover, as all the included studies relied on self-reports to assess the sustainability outcomes, advancing the measurement of sustainability outcomes through robust prospective designs and using the validated purpose designed sustainability assessment tools are also critical.

The longer-term sustainability of CPG adherence (e.g., two or more years after implementation) warrants further investigation. The appropriate timeframe depends on the nature of the implementation guidelines and on what is relevant for the health behaviours studied. A timeframe that is beyond the initial improvement period to provide meaningful data must be chosen when exploring sustainability interventions [47]. Where a long term change is desirable, assessing sustainability longitudinally over several years is essential to capture variations over time (e.g., conceptualizing the dynamic and nonlinear nature of sustainability)
[43]. In addition, most of the included studies aimed to test the effectiveness of the initial implementation innovations without a planned long-term sustainability assessment. Therefore, more studies that include long-term sustainability programs with rigorous multicomponent measures of sustainability are needed, implementation program is meaningful only if program results can be sustained.

\section{Conclusion}

This study advanced the understanding that some implementation strategies may potentially improve the sustainability of healthcare professionals' adherence to CPGs in primary care. Critically, this remains low and unsatisfactory; thereby reducing the potential benefits and impact of CPGs to primary care patients. None of the identified studies applied validated purpose-designed tools to evaluate the sustainability of healthcare professionals' adherence to CPGs. Consequently, there is a great need to develop theory-based or framework-driven interventions with further rigorous research aims to improve this important indicator of evidence-based practice.

\section{Abbreviations}

CPGs: Clinical practice guidelines; PCPs: Primary care providers; RCT: Randomized controlled trial; EPOC: Effective practice and organization of care.

\section{Supplementary Information}

The online version contains supplementary material available at https://doi. org/10.1186/s12875-022-01641-x.

Additional file 1: Supplemental file 1. Suggested risk of bias criteria for EPOC reviews.

Additional file 2. Searching Strategies.

\section{Acknowledgements}

We would like to thank the authors of the included studies who provided additional data and information beyond those described in their publications.

\section{Authors' contributions}

Each author's contributions to the paper are detailed as follows: XLL, IZ, TW, JYT participated in study conception and design, literature searching and sorting, data extraction, analysis and interpretation of data, and paper writing and revising. SS, RC, SE and MJP participated in data analysis and interpretation of data and in revising the paper. All authors read and approved the final manuscript.

\section{Funding}

This review was supported by the Charles Darwin University (CDU) Outstanding Future Researcher Start-up Grant and CDU Institute of Advanced Studies (IAS) Rainmaker Start-Up Grant. Professor Simon Stewart is funded by National Health and Medical Research Council (NHMRC) of Australia [GNT1135894].

Availability of data and materials

All data generated or analysed during this study are included in this published article. 


\section{Declarations}

Ethics approval and consent to participate

Not applicable.

\section{Consent for publication}

Not applicable.

\section{Competing interests}

None.

\section{Author details}

${ }^{1}$ College of Nursing and Midwifery, Charles Darwin University, 410 Ann Street, Brisbane, QLD 4000, Australia. ${ }^{2}$ Torrens University Australia, Wakefield Campus, Adelaide, SA 5000, Australia. ${ }^{3}$ University of Glasgow, Glasgow, Scotland, UK. ${ }^{4}$ Caring Futures Institute, College of Nursing and Health Sciences, Flinders University, Bedford Park, SA 5042, Australia. ${ }^{5}$ Thornlands General Practice, 51 Island Outlook Ave Thornlands, Redland, QLD 4164, Australia. ${ }^{6}$ Cancer \& Palliative Care Outcomes Centre, Faculty of Health, Queensland University of Technology, 60 Musk Avenue, Kelvin Grove, QLD 4059, Australia.

Received: 9 September 2021 Accepted: 15 February 2022 Published online: 01 March 2022

\section{References}

1. Alonso-Coello P, Irfan A, Solà I, Gich I, Delgado-Noguera M, Rigau D, et al. The quality of clinical practice guidelines over the last two decades: a systematic review of guideline appraisal studies. Qual Saf Health Care. 2010;19(6):e58

2. Benzon HT, Joshi GP, Gan TJ, Vetter TR. Development, reporting, and evaluation of clinical practice guidelines. Anesth Analg. 2019;129(6):1771-7.

3. Lugtenberg M, Burgers J, Westert G. Effects of evidence-based clinical practice guidelines on quality of care: a systematic review. BMJ Qual Saf. 2009;18(5):385-92.

4. Ricci-Cabello I, Vásquez-Mejía A, Canelo-Aybar C, de Guzman EN, PérezBracchiglione J, Rabassa M, et al. Adherence to breast cancer guidelines is associated with better survival outcomes: a systematic review and meta-analysis of observational studies in EU countries. BMC Health Serv Res. 2020;20(1):1-12.

5. Lesho EP, Myers CP, Ott M, Winslow C, Brown JE. Do clinical practice guidelines improve processes or outcomes in primary care? Mil Med. 2005;170(3):243-6.

6. Barth JH, Misra S, Aakre KM, Langlois MR, Watine J, Twomey PJ, et al. Why are clinical practice guidelines not followed? Clin Chem Lab Med. 2016;54(7):1133-9.

7. Adams OP, Carter AO. Diabetes and hypertension guidelines and the primary health care practitioner in Barbados: knowledge, attitudes, practices and barriers-a focus group study. BMC Fam Pract. 2010;11(1):1-9.

8. Ament SM, de Groot JJ, Maessen JM, Dirksen CD, van der Weijden T, Kleijnen J. Sustainability of professionals' adherence to clinical practice guidelines in medical care: a systematic review. BMJ Open. 2015;5(12):e008073.

9. Weller CD, Richards C, Turnour L, Team V. Understanding factors influencing venous leg ulcer guideline implementation in Australian primary care. Int Wound J. 2020;17(3):804-18.

10. Damarell RA, Morgan DD, Tieman JJ, Healey DF. Multimorbidity through the lens of life-limiting illness: how helpful are Australian clinical practice guidelines to its management in primary care? Aust J Primary Health. 2021;27(2):122-9.

11. Eccles MP, Mittman BS: Welcome to implementation science. Implementation Sci. 2006;1(1):1-3. https://doi.org/10.1186/1748-5908-1-1.

12. Soicher RN, Becker-Blease KA, Bostwick KC. Adapting implementation science for higher education research: the systematic study of implementing evidence-based practices in college classrooms. Cogn Res Princ Implic. 2020;5(1):1-15.

13. Proctor E, Silmere H, Raghavan R, Hovmand P, Aarons G, Bunger A, et al. Outcomes for implementation research: conceptual distinctions, measurement challenges, and research agenda. Adm Policy Ment Health Ment Health Serv Res. 2011;38(2):65-76.
14. Gruen RL, Elliott JH, Nolan ML, Lawton PD, Parkhill A, McLaren Cl, et al. Sustainability science: an integrated approach for health-programme planning. Lancet. 2008:372(9649):1579-89.

15. Hailemariam M, Bustos T, Montgomery B, Barajas R, Evans LB, Drahota A. Evidence-based intervention sustainability strategies: a systematic review. Implement Sci. 2019;14(1):1-12.

16. Druss BG, Von Esenwein SA, Compton MT, Zhao L, Leslie DL. Budget impact and sustainability of medical care management for persons with serious mental illnesses. Am J Psychiatr. 2011;168(11):1171-8.

17. Berendsen BA, Kremers SP, Savelberg HH, Schaper NC, Hendriks MR. The implementation and sustainability of a combined lifestyle intervention in primary care: mixed method process evaluation. BMC Fam Pract. 2015:16(1):1-12.

18. Kastner M, Sayal R, Oliver D, Straus SE, Dolovich L. Sustainability and scalability of a volunteer-based primary care intervention (health TAPESTRY): a mixed-methods analysis. BMC Health Serv Res. 2017;17(1):1-21.

19. Higuchi KS, Downey A, Davies B, Bajnok I, Waggott M. Using the NHS sustainability framework to understand the activities and resource implications of Canadian nursing guideline early adopters. J Clin Nurs. 2013:22(11-12):1707-16.

20. Maher L, Gustafson D, Evans A. Sustainability model and guide: NHS institute for innovation and improvement; 2009.

21. Cates CJ. Delayed antibiotics for children with acute otitis media: is practice change sustainable? Evid Based Nurs. 2009;12(2):39-40.

22. Enriquez-Puga A, Baker R, Paul S, Villoro-Valdes R. Effect of educational outreach on general practice prescribing of antibiotics and antidepressants: a two-year randomised controlled trial. Scand J Prim Health Care. 2009;27(4):195-201.

23. Gerber JS, Prasad PA, Fiks AG, Localio AR, Bell LM, Keren $R$, et al. Durability of benefits of an outpatient antimicrobial stewardship intervention after discontinuation of audit and feedback. Jama. 2014;312(23):2569-70.

24. Page MJ, Moher D, Bossuyt PM, Boutron I, Hoffmann TC, Mulrow CD, et al. PRISMA 2020 explanation and elaboration: updated guidance and exemplars for reporting systematic reviews. BMJ. 2021;372:n160.

25. Vanselow NA, Donaldson MS, Yordy KD. A new definition of primary care. Jama. 1995;273(3):192.

26. Scheirer MA, Dearing JW. An agenda for research on the sustainability of public health programs. Am J Public Health. 2011;101(11):2059-67.

27. Cochrane Effective Practice and Organisation of Care (EPOC). EPOC resources for review authors: Suggested risk of bias criteria for EPOC reviews. 2017. Retrieved from https://epoc.cochrane.org/resources/epocresources-review-authors.

28. Boutron I, Page MJ, Higgins JPT, Altman DG, Lundh A, Hróbjartsson A. Chapter 7: Considering bias and conflicts of interest among the included studies. In: Higgins JPT, Thomas J, Chandler J, Cumpston M, Li T, Page MJ, Welch VA (editors). Cochrane Handbook for Systematic Reviews of Interventions version 6.2 (updated February 2021). Cochrane. 2021. Available from www.training.cochrane.org/handbook.

29. Spitaels D, Hermens R, Luyten FP, Vandenneucker H, Aertgeerts B, Verschueren S, et al. Educational outreach visits to improve knee osteoarthritis management in primary care. BMC Med Educ. 2019;19(1):66.

30. Presseau J, Mackintosh J, Hawthorne G, Francis JJ, Johnston M, Grimshaw $J M$, et al. Cluster randomised controlled trial of a theory-based multiple behaviour change intervention aimed at healthcare professionals to improve their management of type 2 diabetes in primary care. Implement Sci. 2018;13(1):65.

31. Pinto D, Heleno B, Rodrigues DS, Papoila AL, Santos I, Caetano PA. Effectiveness of educational outreach visits compared with usual guideline dissemination to improve family physician prescribing —an 18-month open cluster-randomized trial. Implement Sci. 2018;13(1):1-11.

32. Wang H-YJ, Ma GX, Liang W, Tan Y, Makambi KH, Dong R, et al. Physician Intervention and Chinese Americans' Colorectal Cancer Screening. Am J Health Behav. 2018;42(1):13-26.

33. van der Velden AW, Kuyvenhoven MM, Verheij TJ. Improving antibiotic prescribing quality by an intervention embedded in the primary care practice accreditation: the ARTI4 randomized trial. J Antimicrob Chemother. 2016;71(1):257-63.

34. Noto H, Tanizawa Y, Aizawa T, Sone H, Yoshioka N, Terauchi Y, et al. Clusterrandomized trial to improve the quality of diabetes management: the study for the efficacy assessment of the standard diabetes manual (SEASDM). J Diabetes Investig. 2016;7(4):539-43. 
35. Martín-Madrazo C, Soto-Díaz S, Cañada-Dorado A, Salinero-Fort MA, Medina-Fernández M, Carrillo de Santa Pau E, et al. Cluster randomized trial to evaluate the effect of a multimodal hand hygiene improvement strategy in primary care. Infect Control Hosp Epidemiol. 2012;33(7):681-8.

36. Trietsch J, van Steenkiste B, Grol R, Winkens B, Ulenkate H, Metsemakers J, et al. Effect of audit and feedback with peer review on general practitioners' prescribing and test ordering performance: a cluster-randomized controlled trial. BMC Fam Pract. 2017;18(1):53.

37. Braithwaite J, Ludlow K, Testa L, Herkes J, Augustsson H, Lamprell G, et al. Built to last? The sustainability of healthcare system improvements, programmes and interventions: a systematic integrative review. BMJ Open. 2020;10(6):e036453.

38. Lennox L, Maher L, Reed J. Navigating the sustainability landscape: a systematic review of sustainability approaches in healthcare. Implement Sci. 2018;13(1):1-17.

39. Hovlid E, Bukve O, Haug K, Aslaksen AB, von Plessen C. Sustainability of healthcare improvement: what can we learn from learning theory? BMC Health Serv Res. 2012;12(1):1-13.

40. Birken SA, Powell BJ, Shea CM, Haines ER, Kirk MA, Leeman J, et al. Criteria for selecting implementation science theories and frameworks: results from an international survey. Implement Sci. 2017;12(1):1-9.

41. Nadalin Penno L, Davies B, Graham ID, Backman C, MacDonald I, Bain J, et al. Identifying relevant concepts and factors for the sustainability of evidence-based practices within acute care contexts: a systematic review and theory analysis of selected sustainability frameworks. Implement Sci. 2019;14(1):1-16.

42. Nilsen P. Overview of theories, models and frameworks in implementation science. In: Handbook on implementation science: Edward Elgar Publishing; 2020.

43. Shelton RC, Cooper BR, Stirman SW. The sustainability of evidence-based interventions and practices in public health and health care. Annu Rev Public Health. 2018;39:55-76.

44. Martin GP, Weaver S, Currie G, Finn R, McDonald R. Innovation sustainability in challenging health-care contexts: embedding clinically led change in routine practice. Health Serv Manag Res. 2012;25(4):190-9.

45. Moore JE, Mascarenhas A, Bain J, Straus SE. Developing a comprehensive definition of sustainability. Implement Sci. 2017;12(1):1-8.

46. de Vries DH, Pool R. The influence of community health resources on effectiveness and sustainability of community and lay health worker programs in lower-income countries: a systematic review. PLoS One. 2017;12(1):e0170217.

47. Stirman SW, Kimberly J, Cook N, Calloway A, Castro F, Charns M. The sustainability of new programs and innovations: a review of the empirical literature and recommendations for future research. Implement Sci. 2012;7(1):1-19.

\section{Publisher's Note}

Springer Nature remains neutral with regard to jurisdictional claims in published maps and institutional affiliations.

Ready to submit your research? Choose BMC and benefit from:

- fast, convenient online submission

- thorough peer review by experienced researchers in your field

- rapid publication on acceptance

- support for research data, including large and complex data types

- gold Open Access which fosters wider collaboration and increased citations

- maximum visibility for your research: over $100 \mathrm{M}$ website views per year

At BMC, research is always in progress.

Learn more biomedcentral.com/submissions 